\title{
Efficiency of diagnostic model to predict recurrent suicidal incidents in diverse world communities
}

\author{
Vatsalya Vatsalya ${ }^{1}$, Kan Chandras ${ }^{2}$, Shweta Srivastava ${ }^{3}$, Robert Karch ${ }^{1}$ \\ ${ }^{1}$ Health Promotion Management American University, Washington D.C., USA; ${ }^{2}$ Behavioral Sciences, Fort Valley State University, \\ Fort valley GA, USA; ${ }^{3}$ Department of Biology, Georgetown University, Washington D.C., USA. \\ Email: vvatsalya@gmail.com
}

Received 16 July 2009; revised 26 August 2009; accepted 27 August 2009.

\begin{abstract}
Suicidal attempts have a very significant effect on the society, and they also reflect on the efforts of the supporting health care and counseling facilities; and the mental health professionals involved. The impact of suicide is further magnified by the needs of persons who attempt suicide multiple times, requiring emergency health care and rehabilitation. Preventing such activities becomes a major task for the support providing agencies as soon as patient with such tendencies are identified. There are repetitive traits that can be observed during the entire therapeutic program among the high-risk group individuals, who are susceptible to this kind of activity and such traits indicate for specific profiling. The aim of the instrument is to prevent the occurrence of the repetitive suicidal attempts of the patients in various world regions, which may have significantly higher and concerning suicide rates. This profile has been constructed on the various parameters recognized in the statistical analysis of the patient population, which have been identified or can be under treatment for their suicidal behavior. This instrument is developed to predict the probability of population segments who may attempt suicide and repetitively, by matching the parameters of the profile with that of the patient pool. Building a profile for the purpose of predicting behavior of this kind can strengthen the intervention strategies more comprehensively and reduce such incidents and health care requirements and expenses.
\end{abstract}

Keywords: Instrument; Parameters; Predictor; Risk Category; Suicidse

\section{INTRODUCTION}

Several world regions face the emerging concern of suicidal incidents and such growth patterns have suggested typical geographical factors playing major contributory roles [1]. Many regions so far have been identified with consistent year wise rising suicide rate and from such locations, Washington State has been included in the study to evaluate this phenomenon and derive a model to investigate and predict suicidal incidents in the general populations. Nationally, Washington State has the $16^{\text {th }}$ highest suicide rate and suicide is the eleventh leading cause of death with 814 death reported in year 2005 with a rate of 13.1 incidents per 100,000 (age adjusted) of population, compared to the national US rate of approximately 11.0 [2]. Suicidal attempts can be five time or more than the suicide death rate [3] (Figure 1).

There have been consistencies in reports that even after getting initial medical and other support services, patients attempt suicide again in Washington State with similar rate as without the interventions [4]. During 2003-2005, males in Washington accounted for 79\% of completed suicides, with the highest rate of suicide, observed in the group among the age group of 75 years and more [5]. In 2004; in the 15-24 age groups, suicide was the second most leading cause of death and altogether 17\% deaths [6]. Nearly two-thirds of teens with clinical depression go unnoticed and may not get treated; among these, males 15 to 19 years old are five times more likely

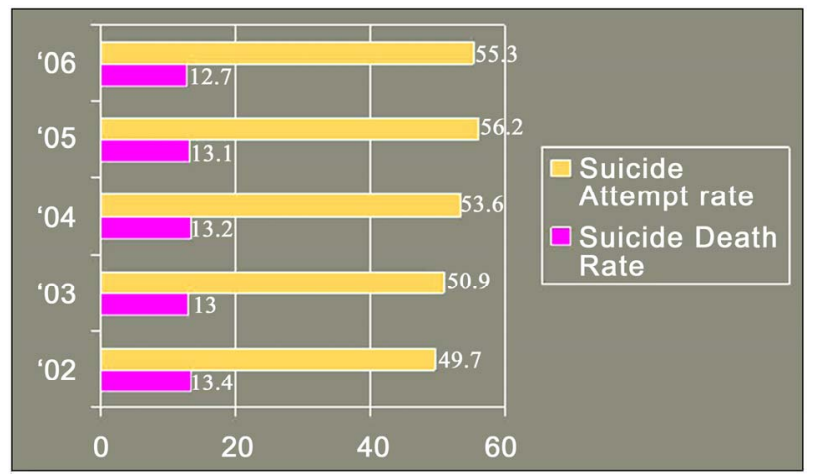

Figure 1. Yearly suicide rate representation per 100,000 populations (Suicide attempt and death rate versus years as x:y coordinates). 


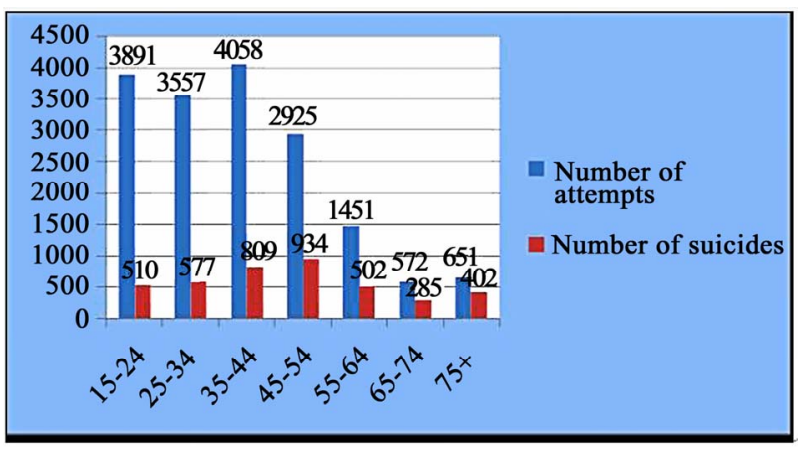

Figure 2. Age-group based comparisons of number of suicidal incidents (Age-groups versus number of incidents as $\mathrm{x}$ :y coordinates).

than females to complete suicides [7] (Figure 2). The frequency of suicide attempts can increase, if the causative factors are not resolved. Extent for emotional and physical pain cannot be assessed for individuals and their relatives, though approximately three billion US dollars of loss associated with the suicidal incidents has been recorded so far during the year 2002-2006 [8]. Preventive procedures have been particularly promoted to reduce such incidences, which have priority concern to precisely identify target population. Development of a predicting instrument, which can provide appropriate behavioral evaluation, can be one of the choices to this requirement.

This instrument is based on set of primary repetitive characters, which can provide a predictable analysis of such behavior among the high-risk group individuals, who are susceptible to commit or attempt suicide. The concept of predictor is to identify the patients with such tendency or ideations and inform the healthcare profes- sionals and people concerned, to prepare and implement specific therapeutic plan to minimize such incidents further. A predictor profile for the purpose of interpreting specific behavior would strengthen the intervention strategies, reduce the frequency of such incidents, and regularize involvement of health care and financial resources.

\section{METHODOLOGY}

Data source for the profile analysis has been collected from yearly published hospitalization reports of suicide incidents from Washington State public health statistical data bank, Washington State Injury and Violence Prevention Program, 2008; and GIS from 2002 to 2006 year. Data from 15,826 cases with suicides and single or multiple identified attempts of history during the interval 2002-2006, between the age group of 15 and 75 + years, have been included in the study (Figure 3). Significant cohort parameters have been studied from the target population for the development of the instrument, namely gender, age groups, health condition, biological and environmental factors (namely economic, academic, social, ethnic and likewise). Statistical analysis has been performed to identify repetitive characteristics of the parameters with annual rate and frequency comparisons. An analog model is constructed utilizing the parameters and their characteristics under observations. The characteristics have been broadly grouped as parameters of interests and highly repetitive prevalent characteristics observed in the evaluation have been consigned under high risk category (Colored in red, Figure 6); average repeating characteristics as intermediate and less frequently occurring as low risk category. These parameters were constructed in the form a pathway with the promi-

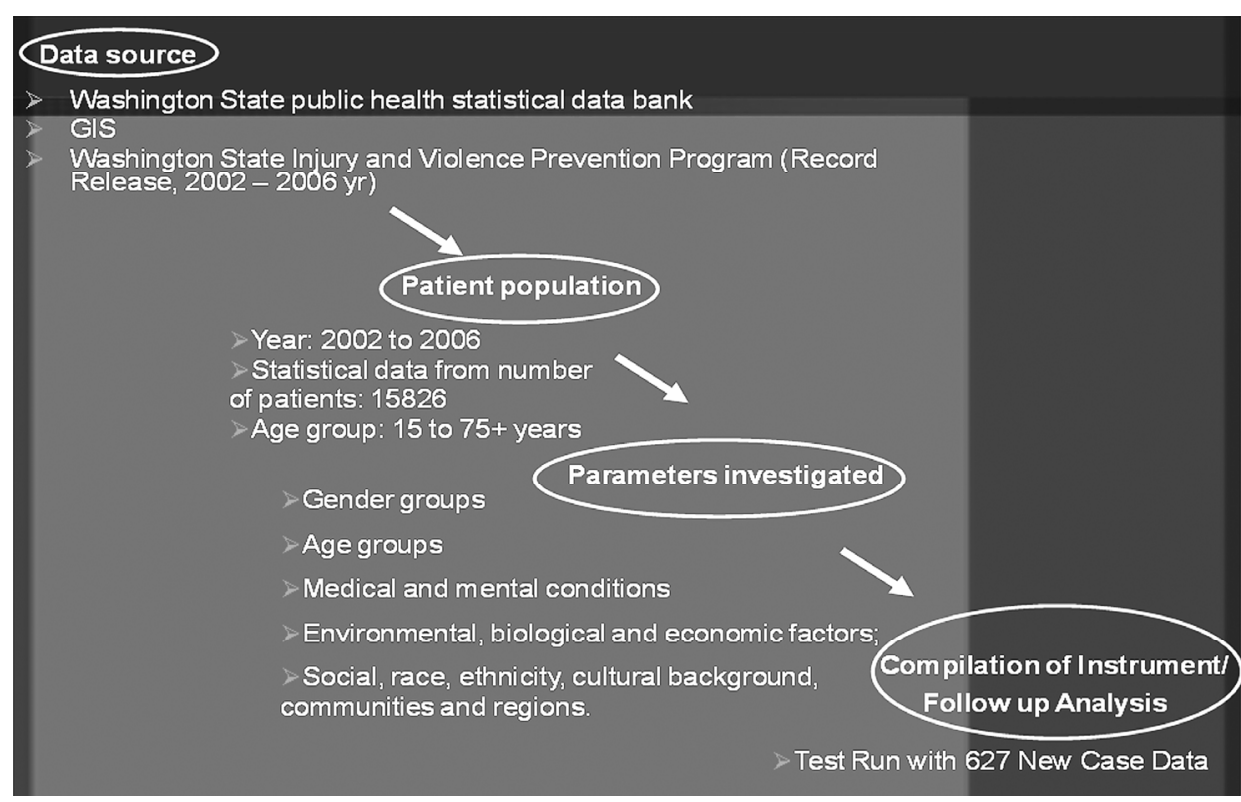

Figure 3. Methodology illustration for investigating model parameters. 
nent parameters evaluated initially within which all the characteristics of each parameter undergo comparison for in track corresponding profile similarities.

Characteristics which have not demonstrated significant prevalence in the analysis or are not significantly identifiable in the data search and evaluations have not been included in the development of the analog at this stage of the study. A follow up evaluation assessment has been conducted for comparing this profile with the data from new reports to evaluate the predictability the evaluations and to develop the validity of the model.

\section{RESULTS}

The parameters are assessed in relation to the numbers of attempts and actual suicide incidents reported between year 2002 and 2006. The age groups 15-24 and 35-44 yrs have the maximum incidents of attempted suicides (Figure 2).

The leading conversion of the attempts into actual suicide is demonstrated by the age group 45-54 yrs and from 2002 year onwards, there is a steady increase in the rate of suicide and suicide attempts in this age group (Figure 4). Males in Washington accounted for 79\% of total suicides, though majority of females have attempted suicides with a rate of $62 \%$. There has been a consistent annual rise in the number of suicidal attempts as evident from annual health reports (Figure 5). Suicide comparison ratio among the adult male and female patient population rate has been measured as $7: 2$, which is evident from the finding that during 2003-2005 period. Among adolescent and young age groups, approximately $25 \%$ of attempted suicide by youth male results in death; whereas three-four percent deaths result in females. 15 to 19 year old males are five times more likely than females to commit suicides. Caucasian and American Indians demonstrate largest proportion of actual number of suicidal attempts and incidents as 14 every 100,000 resident population each.

Data reflects that in Caucasian and Native Indian groups, suicide is occurring with 14 per 100,000 rates each though African-American, Asian and Hispanic groups demonstrated non-significant rate of eight, eight and six consecutively. High competition, economic and social hardship can be observable factors among Caucasian though relationship cannot be established directly; partially available particulars are only confirmed presently for family predisposition, abuse, addiction and health concerns among with Native Americans too [9]. Suicide rate as observed in relation with academic background can be described with the ratio of 28:14:9 with 28 with patients having $12^{\text {th }}$ grade or less education, 14 with some college level programs and 9 with graduation or post graduation. Linear regression of suicide rate has been observed with progression of academics in the statistical evaluation. Poisoning is identified as the most common means for attempted suicide cases among 15-24 and 35-54 age group whereas firearms are as the most common means among the young and adult groups for suicide deaths. Based on the results of statistical analysis and evaluations, several parameters and their characteristics have been identified and incorporated for the development of the model for the instrument with the order as gender, age group, ethnicity, health, education, environmental condition, and; exposure and access to perform self-fatality for the Washington region (Figure 6).

The construction of the analog utilizes major and recurring parameters first and tracks subsequent parameters and their characteristics in the descending order of evaluation. These Follow-up evaluations with parameters evaluated from various new case studies (a total of 627 case data) randomly, indicated results in compliance with an accuracy of more than $90 \%$ of the total cases, when the parameters are run through the analog model.

\section{DISCUSSION}

Age groups 15-24 and 35-54 year have demonstrated the majority of incidents of suicide and suicidal attempts. Parallel emergence of economic hardship can be projected as one of the reasons for the growth of suicide rate among the 45-54 year age group [10].

Higher suicide rate among is recorded among males, approximately $25 \%$ of attempted suicide by youth male results in death, whereas 3-4\% death results in females, though higher attempt rate among females is observed [11]. Health conditions (depression, substance abuse,

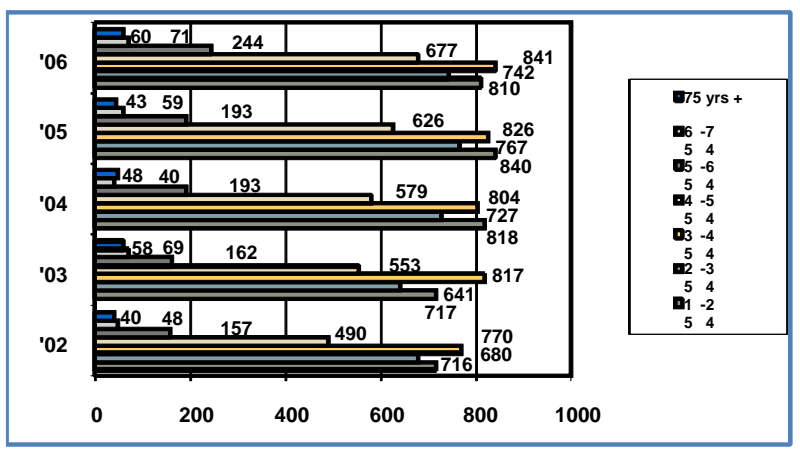

Figure 4. Yearly distribution of suicidal incidents in various age-groups (Number of incidents versus years as $\mathrm{x}$ : $\mathrm{y}$ coordinates).

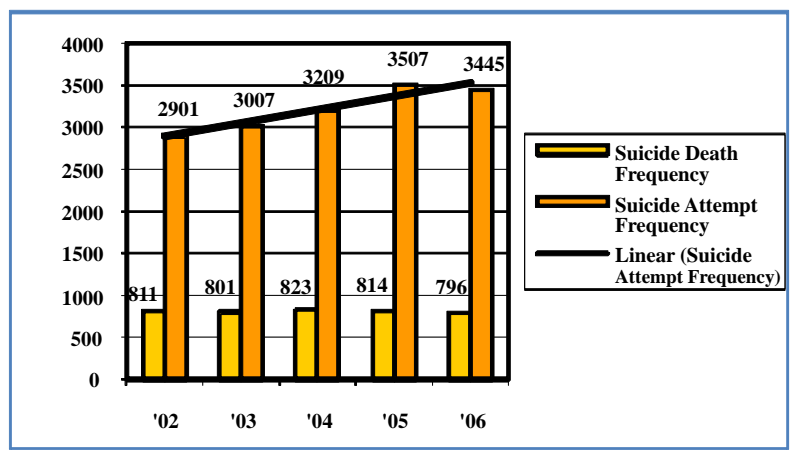

Figure 5. Yearly distribution of total number of suicidal incidents (Years versus number of incidents as x:y coordinates). 


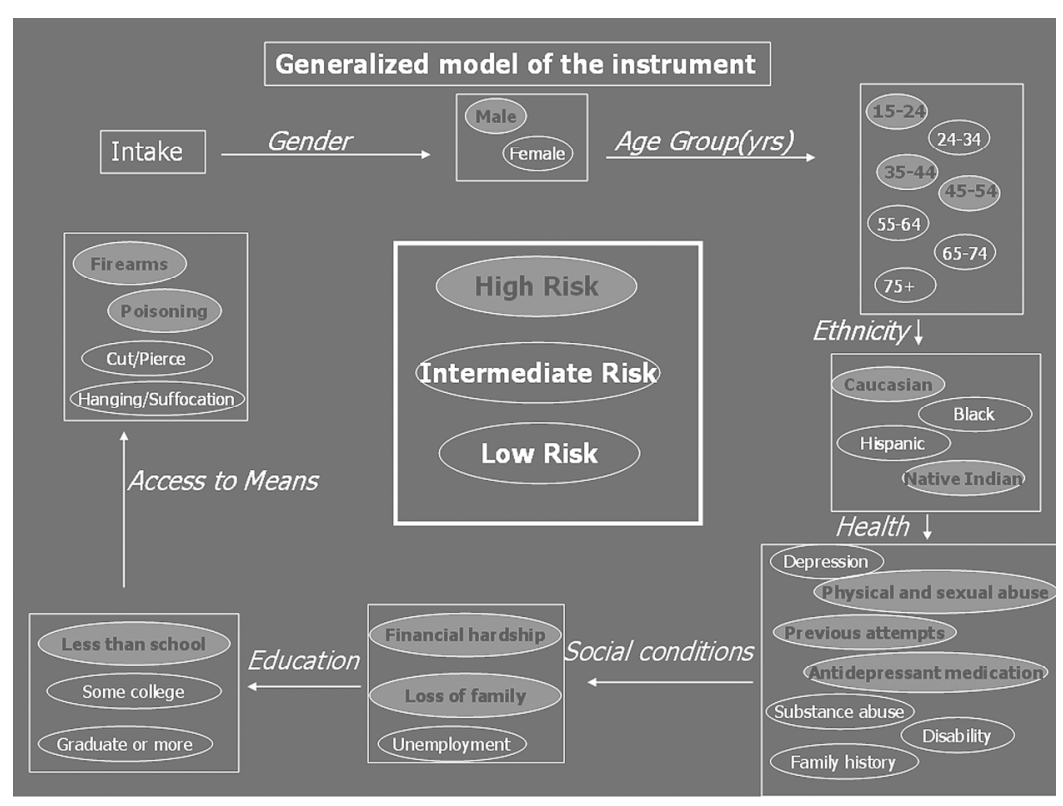

Figure 6. Analog construction of diagnostic model.

ideation and conditions like family history, sexual orientation and abuse, disability) show higher probability of suicidal incidents (Figure 7). Individuals with physical and sexual abuse have been observed to have four times higher suicide attempt rate than non-abused. Among African American communities, some of the major factors are community segregation, economic hardship and access to weapon; though cultural encapsulation and stress is prevalent among other minority groups. From 2002 year onwards, there is a steady increase yearly, in the number and rate of suicide incidents in the 35-54 age groups [12]; and the proportion of people living in poverty increased, though data is not sufficient enough to identify relation to economic hardship [13]. Post-Traumatic Stress Disorder, economic hardship, social constructs are being studied as an extension of the ongoing study.

\section{CONCLUSIONS}

The complexity of suicidal behavior and ideation requires multiple and immediate preventive approach and application of a predictor model can supplement targeting population, requiring adequate health care services to prevent suicide. Since much published material and clinical experience demonstrate a number of causative factors associated with suicide, the early identification and appropriate treatment of this condition is an important strategy for prevention. Improvements may be required for broadening data collection and investigatory techniques for possible suicidal attempts, social constructs and economic causes; biological and environmental factors and minority groups from both fatal and

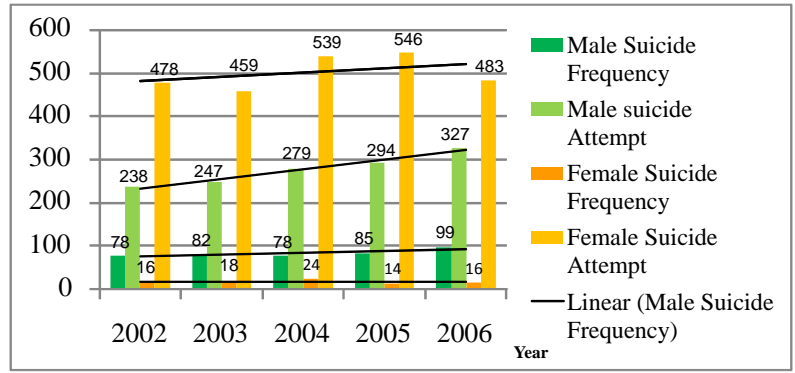

Figure 7. Yearly distribution of depression-originated youth suicidal incidents (Years versus number of incidents as $x: y$ coordinates).

non-fatal suicide incidents. Utilization of the instrument at the key regions and community level; significantly social and community based agencies, academic institutions and care centers can be highly valuable and recommended. Design, planning and Implementation of intervention strategies for newly growing suicidal incidents in the 45-54 year age group should be focused appropriately. A preliminary investigation in various world regions with above-average suicide rate have identified points of interests namely Kutznetsk Basin, former USSR republics (between 1980 and 1995 citing economic instability); among indigenous peoples in both Australia and Canada in the last 20 years [1]. Depending upon the various factors and parameters in different world locations, with specific standardization, an appropriate construction of the predictor model and evaluation may be utilized for similar diagnostic procedures and further research may provide essentials and scope for development for area specific revisions. 


\section{REFERENCES}

[1] World Report on Violence and Health: Self-Directed Violence (1999). Geneva, Wold Health Organization (document WHO/ HSC/PVI/99.11).

[2] Injury and Violence Prevention Program. Washington State Department of Health [online], 2007, http://www. doh.wa.gov/hsqa/emstrauma/injury/data-tables.

[3] Washington State Department of Health, (2004). Suicide chapter, The Health of Washington State 2004 Supplement. Olympia, WA, http://www.doh.wa.gov/HWS/HWS 2004supp.htm.

[4] American Journal of Psychiatry [online], 2006, http://ajp.psyh.iatryonline.org/cgi.

[5] Health of Washington State Report. Washington State Department of Health [online], 2007, http://www.doh.wa.gov/HWS/default.htm.

[6] Simon, G. E., Savarino, J., Operskalski, B., and Wang, P. S., (2006) Suicide risk during antidepressant treatment, Am. J. Psychiatry, 163, 41-47

[7] Center of Health Statistics (Death data). Washington State Department of Health [online], (2007), http://www. doh.wa.gov/ehsphl/chs-data/death/dea_VD.htm.

[8] Center of Health Statistics (Hospital data). Washington State Department of Health [online], (2007), http://www. doh.wa.gov/EHSPHL/hospdata/default.htm.

[9] Mayer-Gross, W., Slater, E., and Roth, M., (1960) Clinical Psychiatry, London, Cassell.

[10] U.S. Centers for Disease Control and Prevention, National Centers for Injury Prevention and Control. (2007). Web-based Injury Statistics Query and Reporting System (WISQARS), Retrieved January 3, 2007 from http://www. cdc.gov/ncipc/wisqars.

[11] Rotheram-Borus, M. J., Piacentini, J., Cantwell, C., Beline, T. R., and Sone, J. (2000). The 18-month impact of an emergency room intervention for adolescent female suicide attempters. Journal of Counseling and Clinical Psychology, 68(6), 1081-1093.

[12] Centers for Disease Control and Prevention (CDC). Web-based Injury Statistics Query and Reporting System (WISQARS) [Online], (2005) National Center for Injury Prevention and Control, CDC (producer), Available from http://www.cdc.gov/ncipc/wisqars/default.htm.

[13] Geographic Information Systems. Division of Information Resource Management, Washington State Department of Health [online], 2007, http://www.doh.wa.gov/gis/gisdata.htm. 